\title{
Distribution of Sodium Cations in Faujasite-Type Zeolite: A Canonical Parallel Tempering Simulation Study
}

\author{
Christèle Beauvais, Xavier Guerrault, François-Xavier Coudert, Anne Boutin, and \\ Alain H. Fuchs* \\ Laboratoire de Chimie Physique, UMR 8000, CNRS, Université de Paris-Sud, 91405 Orsay, France
}

Received: July 17, 2003; In Final Form: October 8, 2003

\begin{abstract}
A Monte Carlo replica exchange (MCRE) algorithm was used to compute the sodium cation distribution in bare faujasite zeolite with a number of cations per unit cell ranging from zero $(\mathrm{Si}: \mathrm{Al} \rightarrow \infty)$ to $96(\mathrm{Si}: \mathrm{Al}=1)$. Eight independent realizations of the system were simulated simultaneously, in the temperature range 300$2325 \mathrm{~K}$. The resulting distributions at room temperature were found to be in very good agreement with both available experiments and the analytical quasi-chemical model of Mortier and co-workers. A single canonical simulation at room temperature using site-to-site hopping yields identical results. The main advantage of the MCRE method is that no assumption is needed on the actual cation adsorption sites. One could thus, in principle, predict cation locations and distributions in a nanoporous solid for which the precise location of extraframework cations is not known.
\end{abstract}

\section{Introduction}

Aluminosilicate faujasite-type zeolites have been widely studied in the past few years, owing to their industrial importance in gas adsorption and separation. In these materials, the presence of aluminum atoms introduces charge defects that are compensated with some nonframework cations (sodium, potassium, barium, etc.). Adsorption properties in zeolites are closely related to the location of nonframework cations and to their accessibility to adsorbed molecules. However, the precise location and the distribution of monovalent cations in faujasite by conventional diffraction techniques is rendered especially difficult because of partial occupancy affecting low symmetry sites.

Since the initial diffraction work by Mortier, ${ }^{1}$ there have been a significant number of studies dedicated to the reinvestigation of cation location in dehydrated $\mathrm{NaY}$ and $\mathrm{NaX}$, using powder or single-crystal diffraction refinements ${ }^{2-17}$ or NMR studies. ${ }^{15,18-21}$ Theoretical models ${ }^{10,22-27}$ and computational approaches have also been used to predict cation location in this material ${ }^{13,28-31}$ as well as in other zeolites. ${ }^{48-50}$

The cation distribution is usually described as follows (see Figure 1). $\mathrm{Na}^{+}$can occupy sites I, located in the hexagonal prisms which connect sodalite cages. Sites I' are inside the sodalite cages facing sites I. Sites II are in front of the 6-rings inside the supercages. Sites III are also in the supercages, near the 4-rings of the sodalite cages. Site I has a multiplicity of 16 per unit cell, sites I' and II have a multiplicity of 32 , and site III has a multiplicity of 48 per unit cell. Some cations have also been found (in the case of $\mathrm{NaX}$ ) in the 12-ring windows. ${ }^{12-13}$ These are called sites III'. Sites III and III' are believed to be of higher potential energy than sites I, I', and II. At low occupancy ( $\mathrm{Si}: \mathrm{Al} \geq 2$ ), cations are known to occupy sites I, I', and II only.

Grand canonical Monte Carlo simulations, using statistical bias for studying large anisotropic molecules, have been shown to provide reasonably accurate predictions of adsorption data. ${ }^{32}$

* To whom correspondence should be adressed. E-mail: alain.fuchs@ lcp.u-psud.fr.

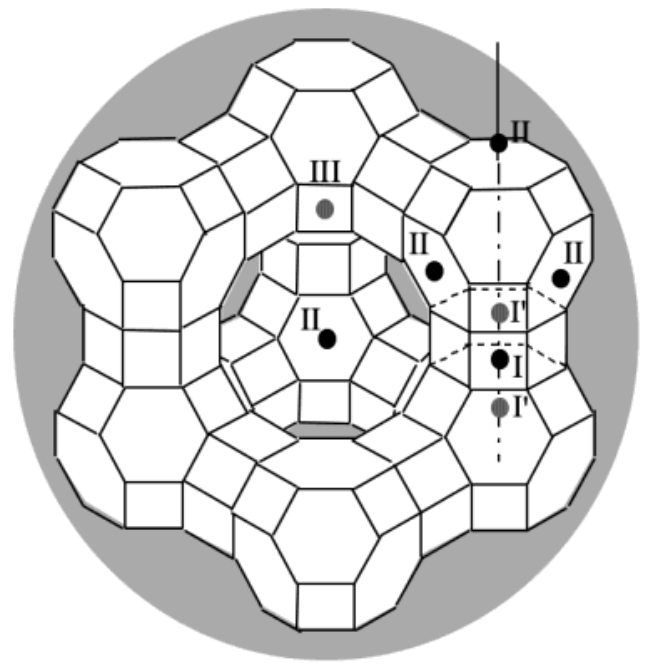

Figure 1. Schematic view of a faujasite supercage with the site I, I', II, and III locations.

Molecular simulation is a method of choice for direct predictions of adsorption selectivity in mixtures, using experimentally obtained pure components data to fit some of the potential parameters. As pointed out by $\mathrm{Talu}^{33}$ the pure component isotherm is the simplest measurement of adsorption equilibrium and it can be determined quite accurately. On the other hand, binary mixture measurements are at least an order of magnitude more complicated and time-consuming. It is thus suggested that a combination of experiments and simulations can be used to help in the rational design of adsorbents for mixture separations. To achieve this goal, there is a need for some refinements of the simulation models together with some progress in the simulation methods.

Here we describe a method that enables one to take into account in the Monte Carlo simulations the possible redistribution of extraframework cations upon adsorption of guest molecules. In most of the previously published computational studies of adsorption, cations were placed in the positions 
obtained experimentally for the dehydrated material and kept fixed throughout the simulations. ${ }^{32}$ Only recently have the cation positions been taken into account in the simulation of alkane mixture adsorption in low aluminum content ZSM-5. ${ }^{34}$ The need clearly exists for a method that could enable one to predict the way cations are distributed among the available sites in systems for which experiments are lacking and how cations redistribute upon adsorption of highly polar molecules. Some experimental evidence exists for cation redistribution upon $\mathrm{HFC}^{35}$ and water ${ }^{36}$ adsorption in faujasite. This was confirmed by a recent molecular dynamics study of some $\mathrm{HFC}\left(\mathrm{CF}_{2} \mathrm{H}-\mathrm{CF}_{2} \mathrm{H}\right.$ and $\mathrm{CH}_{2} \mathrm{~F}-\mathrm{CF}_{3}$ ) in $\mathrm{NaX}^{37}$

In a previous paper, ${ }^{31}$ we have shown that the extraframework sodium cations in faujasite experience an extremely rough potential energy landscape. Numerous metastable states were obtained either through standard Monte Carlo simulations or simulated annealing. In many cases, we have found it necessary to combine simulated annealing with long distance site-to-site hopping in order to reach the (supposedly) most stable state corresponding to the most probable cation distribution for a given Si:Al ratio.

Here we use the canonical replica-exchange Monte Carlo method $^{38-42}$ (also known as parallel tempering), to enhance the configuration sampling of low-energy states in the sodiumfaujasite system. In this method, several independent realizations of the system are simulated simultaneously, each differing in temperature. As the simulation proceeds, systems at adjacent temperatures are allowed to interchange configurations from time to time, subject to a specific acceptance criteria. These swap moves considerably improve the sampling of configuration space. The higher temperatures are chosen in such a way that the system can easily overcome energy barriers and thus can provide the low-temperature systems with configurations that cover a broad range of configuration space. This method was used here to compute the stable distributions at room temperature for a number of cations per unit cell ranging from 0 ( $\mathrm{Si}: \mathrm{Al} \rightarrow$ $\infty)$ to $96(\mathrm{Si}: \mathrm{Al}=1)$.

The remainder of the paper is organized as follows. In section 2 , we discuss the computational methodology. In section 3 , we present the results of the replica-exchange Monte Carlo simulations and we compare our results with both the available experimental data and the analytical model proposed by Mortier and co-workers. ${ }^{10,25-27}$ Concluding remarks are given in section 4.

\section{Computational Methodology}

A. Simulation Model. The faujasite model, atomic charges, and the cation force field used have been described in details in a previous paper. ${ }^{30}$ The framework structure of dehydrated faujasite was taken from the experimental neutron diffraction studies of Fitch et al. ${ }^{4}$ The crystalline structure is described in the $F d 3 m$ space group, and the cubic lattice parameter is 24.8536 $\AA$. The same framework structure was used throughout this work and was considered as rigid. One unit cell of faujasite was used as the simulation box, with periodic boundary conditions in all three directions. The different host framework-guest cations systems had a $\mathrm{Si}$ :Al ratio ranging from 1 to $\infty$. The corresponding number of sodium cations per unit cell ranged from 96 ( $\mathrm{Si}$ : $\mathrm{Al}=1)$ to $0(\mathrm{Si}: \mathrm{Al} \rightarrow \infty)$. For each system, an average tetrahedrally bonded $(\mathrm{T})$ atom was used, for reasons detailed in ref 30. Partial charges on the oxygen and $\mathrm{T}$ atoms were adjusted in the way described in ref 30, using the electronic structure calculations of Mortier and co-workers, ${ }^{43}$ and the number of cations was fixed accordingly. Sodium cations bear a charge of $+1 \mathrm{au}$.

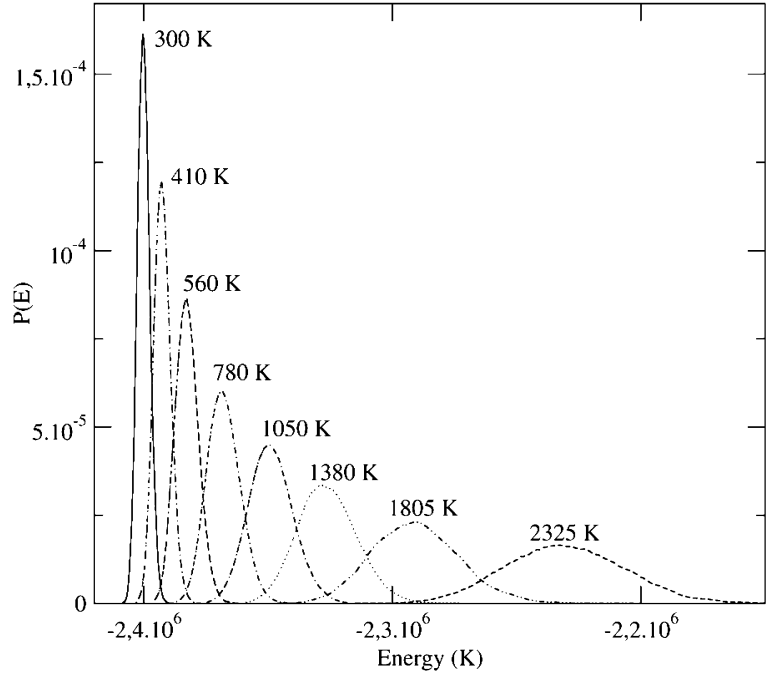

Figure 2. Potential energy distribution for the eight realizations of the system.

The cation force field has been adapted from the work of Jaramillo and Auerbach, ${ }^{29}$ in the way described in ref 30 . The cation-framework potential consists of an exp-6 repulsiondispersion term that acts between the cation and the oxygen atoms of the faujasite and a Coulombic term that acts between the cation and both the oxygen and $\mathrm{T}$ atoms of the framework. Sodium cations interact with each other through a single Coulombic term. Different cation force fields have been tested in a previous work, and it turned out that the one proposed by Jaramillo and Auerbach was doing the best job in reproducing the cation positions in NaY. ${ }^{44}$ Finally, Ewald sums were used to calculate the long-range Coulombic terms. The Ewald parameter $\alpha$ was equal to $0.19 \AA^{-1}$ and the $k$ vectors were such that $k \in[-3,3]$. This allows us to reproduce the exact Coulombic energy with high precision ( $<1 \%$ error).

B. Monte Carlo Simulations. We performed replicaexchange canonical Monte Carlo simulations of the sodium cations subjected to the external field imposed by the rigid faujasite framework host. For each system (corresponding to a given $\mathrm{Si}$ :Al ratio), eight independent realizations of the system were simulated simultaneously. The chosen temperatures (in Kelvin) were 300, 410, 560, 780, 1050, 1380, 1805, and 2325. The highest temperature was chosen in such a way that the cations experienced a "liquidlike" behavior. The lowest temperature is room temperature, at which most of the adsorption experiments have been carried out. It was found that eight different temperatures were needed in order to ensure a reasonable degree of overlap between the potential energy distributions of neighboring state points (Figure 2). This number may seem quite large, but one has to remember that a unit cell of faujasite is made of $576 \mathrm{~T}$ and oxygen atoms, plus a variable number of cations (from 0 to 96). The number of realizations needed to ensure an effective simulation depends on the size of the system, since the width of the probability distribution function decreases with the system size. Adjacent temperatures are in a ratio of $\sim 1.3$ for each temperature interval. It has been shown recently by $\mathrm{Kofke}^{45}$ that the acceptance probability can be made uniform across the multireplica partition by selecting adjacent temperatures that are in a fixed ratio.

Each simulation ranged between $10^{7}$ and $3 \times 10^{7}$ Monte Carlo trials. In each trial, either a cation displacement or a replica exchange was attempted, selected at random, such that displacements were attempted 100 times more often than exchanges. To satisfy detailed balance, the displacement and exchange trials 

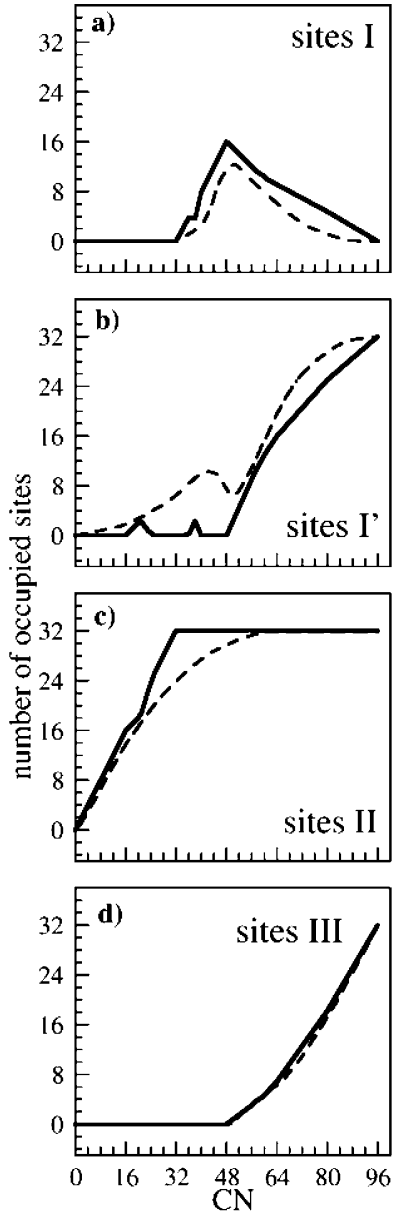

Figure 3. Sodium cation occupancy of sites I (a), sites I' (b), sites II (c), and sites III (d). Full line: parallel tempering simulations. Dotted line: QC model.

were accepted with probabilities

$$
\begin{gathered}
P_{\mathrm{acc}}^{i \rightarrow j}=\min \left[1, \exp \left(-\beta\left(U_{j}-U_{i}\right)\right)\right] \\
P_{\mathrm{acc}}^{m \rightarrow n}=\min \left[1, \exp \left(-\left(\beta_{m}-\beta_{n}\right)\left(U_{n}-U_{m}\right)\right)\right]
\end{gathered}
$$

for the displacement and exchange trial, respectively. In eq 1 , $i$ and $j$ refer to two successive configurations in a canonical simulation for a given realization at a temperature $T=1 / k_{\mathrm{B}} \beta$ ( $k_{\mathrm{B}}$ is the Boltzmann's constant). For the exchange trial, we consider a pair of systems (subscripts $\mathrm{m}$ and $\mathrm{n}$ ) at temperature $T_{\mathrm{m}}=1 / k_{\mathrm{B}} \beta_{\mathrm{m}}$ and $T_{\mathrm{n}}=1 / k_{\mathrm{B}} \beta_{n}$ respectively.

\section{Results and Discussion}

A. Cation Distributions from Parallel Tempering Simulations. Sodium cation occupancy of sites I, I', II, and III as a function of the cation number per unit cell at $300 \mathrm{~K}$ are shown in Figure $3 a-d$. The dotted line shows the prediction of the analytical "Quasi-Chemical" model of Mortier and coworkers, ${ }^{10,25-27}$ which we will discuss in the next section.

In Figure $4 a-d$, the simulation data and the quasi-chemical model are compared with the available experimental data, in the range of 48-96 cations per unit cell. The overall agreement is quite satisfactory. Some experimental data can be found for cation numbers lower than 48 . However, these experiments ${ }^{10,26}$ have actually been performed on NaHY samples, in which sodium cations were progressively exchanged by $\mathrm{H}$ atoms, at a
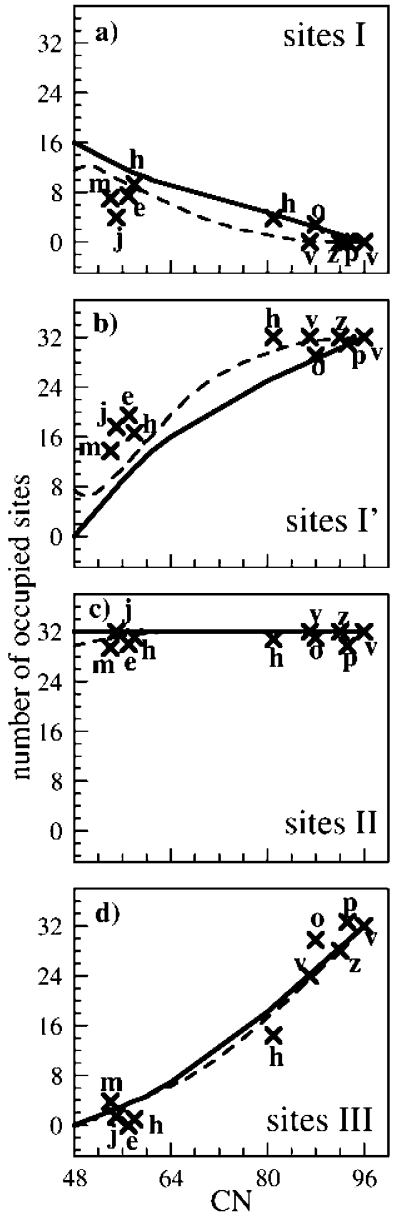

Figure 4. Sodium cation occupancy of sites I (a), sites I' (b), sites II (c), and sites III (d) from $\mathrm{CN}=48-96$. Full line: parallel tempering simulations. Dotted line: QC model. Crosses: experiments. The letters correspond to the following references: $m$ (ref 46), j (ref 3), e (ref 2), $\mathrm{h}(\operatorname{ref} 7), \mathrm{z}(\operatorname{ref} 16), \mathrm{p}(\operatorname{ref} 17), \mathrm{v}(\operatorname{ref} 13)$, and o (ref 12).

fixed Si:Al ratio. The corresponding cation distributions cannot be compared to our simulations.

The observed trends in the simulated site populations can be understood in terms of a competition between the individual cation-framework potential energy interaction and the Coulombic interaction between cations. In Figure 5 is shown the cationframework potential energy in each site (regardless of the cation-cation interaction), as a function of the cation number. At low cation number $(\mathrm{CN})$, site II is the most favorable adsorption site. This explains why cations preferentially occupy sites II from $\mathrm{CN}=0$ to approximately 20 .

Above $\mathrm{CN} \sim 20$, site $\mathrm{I}^{\prime}$ is becoming (individually) more stable than site II. Some site I' occupancy is then observed in the range $20<\mathrm{CN}<30$ (Figure 3b), but the overall tendency is to keep filling sites II up to a complete occupancy of 32 cations per unit cell. It turns out that the Coulombic cation-cation interaction, while negligible in the limit of $\mathrm{CN} \rightarrow 0$, becomes progressively dominant with respect to the cation-framework interaction as $\mathrm{CN}$ increases. Thus, instead of filling sites $\mathrm{I}^{\prime}$, cations will keep occupying sites II because the II-II distance is larger than the II- $\mathrm{I}^{\prime}$ one (see Figure 1).

By the time sites II are completely occupied $(\mathrm{CN}=32)$, cations start to fill sites I. Again, the dominant term in the total interaction energy is now the cation-cation Coulombic energy. Thus, the system prefers to occupy sites I, which are further away from sites II than sites I' are from site II. This holds up to a complete occupancy of 16 sites I per unit cell. 


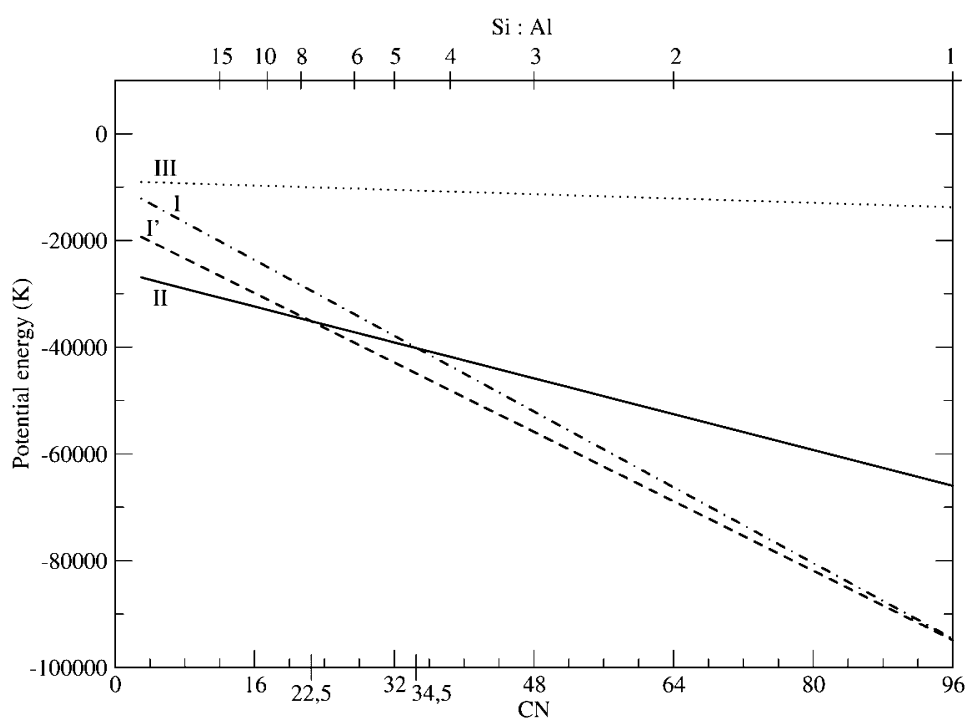

Figure 5. Cation-framework potential energy as a function of the cation number $\mathrm{CN}$ for site I, I', II, and III.

Above $\mathrm{CN}=48$, which corresponds in our model to a complete occupancy of sites II and I, a further increase in $\mathrm{CN}$ can only be achieved by occupying sites I'. Because the distance between sites I and I' is extremely small (2.18 $\AA$ ), a sodium cation can only occupy a site $\mathrm{I}^{\prime}$ if the adjacent cation in site I is at the same time displaced toward the opposite site $\mathrm{I}^{\prime}$, due to the strong repulsive Coulombic interionic energy (see Figure 1). This explains the simultaneous increase in the site $I^{\prime}$ population and decrease in site I occupancy. We note in passing that it has been supposed for long, and checked experimentally, that adjacent sites I and I' are not simultaneously occupied. ${ }^{24}$

Above $\mathrm{CN} \sim 56$, a progressive occupation of sites III is observed. In much the same way as in our previous work, ${ }^{30}$ the positions spontaneously reached by the cations after convergence of the replica-exchange $\mathrm{MC}$ runs at $300 \mathrm{~K}$ display some small changes with respect to the crystallographic sites taken from the works of Fitch $^{4}$ and Mellot. ${ }^{11}$ While site II location is unchanged, two extra locations are observed for sites I' and III, respectively. The extra site $\mathrm{I}^{\prime}$ is located at $\sim 0.2 \AA$ away from the initial site $\mathrm{I}^{\prime}$ toward to hexagonal prism which leads to broaden the cation distribution around site $\mathrm{I}^{\prime}$. Two types of site III are observed in the simulations. The first one lies in the middle of a 4-ring. The second one is close to the 12-ring window. It corresponds quite well to the site III' location reported by Vitale et al. ${ }^{13}$ The relative occupancy of sites III and III', however, is not satisfactorily reproduced in the simulations. A better agreement with experiments has been obtained recently by using a model in which silicon and aluminum atoms are explicitly distinguished, instead of using an average $\mathrm{T}$-atom. ${ }^{31}$ The reader is referred to ref 30 and 31 for more details on this issue.

B. Comparison with the Analytical "Quasi-Chemical"

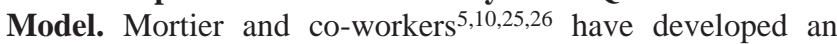
analytical model for cation distribution in faujasite-type zeolites, which was called the "quasi-chemical model" (QC). In this model, only interactions between cations in nearest neighbor sites (I and $\mathrm{I}^{\prime}$ ) are taken into account. A cation in site II is considered to have no neighbor (or to behave ideally). A single potential energy of interaction $(-w)$ is postulated for any nearest neighbor pair. The canonical partition function of the system is written in terms of the number of nearest neighbor pairs configurations, which van Dun et al., ${ }^{25}$ given the assumptions of the model, have been able to explicitly enumerate. The site populations can then be expressed as a function of three parameters: the interaction energy $w$ and the energy level differences $\left(e_{\mathrm{I}^{\prime}}-e_{\mathrm{I}}\right)$ and $\left(e_{\mathrm{II}}-e_{\mathrm{I}}\right)$, where $e_{\mathrm{i}}$ denotes the stabilization energy of the cation at site i relative to the groundstate energy at infinity.

Using the data of a $\mathrm{Na}_{x} \mathrm{HY}$ series of structures, a best fit for these three parameters was determined. ${ }^{25}$ Furthermore, the energy differences were assumed to vary linearly with the aluminum content of the faujasite framework. ${ }^{10}$ The values for the QC model parameters were then

$$
\begin{gathered}
e_{\mathrm{I}^{\prime}}-e_{\mathrm{I}}=-38.23 \mathrm{~kJ} \mathrm{~mol}^{-1}+0.93 N_{\mathrm{Al}} \\
e_{\mathrm{II}}-e_{\mathrm{I}}=-45.23 \mathrm{~kJ} \mathrm{~mol}^{-1}+0.93 N_{\mathrm{Al}} \\
w>22 \mathrm{~kJ} \mathrm{~mol}^{-1}
\end{gathered}
$$

where $N_{\mathrm{Al}}$ is the number of aluminum atoms per unit cell of faujasite, which is equal to the number of cations in $\mathrm{NaY}$ or $\mathrm{NaX}$. The observed trends of the cation distributions in most $\mathrm{X}$ and Y faujasites were found to be well reproduced by the QC model. ${ }^{10}$ In Figure 3, we have sketched the site populations as a function of the cation number $\mathrm{CN}$, using the QC model with $w=50 \mathrm{~kJ} \mathrm{~mol}^{-1}$, together with the results of the present parallel tempering simulations. We note in passing that the QC model takes explicitly into account three sites only (I, I', and II). The site III population shown in Figure 3 corresponds to cations which have been repelled from the $\mathrm{I}-\mathrm{I}^{\prime}$ configuration. Once sites I' and II are fully occupied, all of the other cations (as $\mathrm{CN}$ increases) are accommodated in site III.

The overall agreement between the simulations and the QC model is quite good. The increase in site II population is steeper in the MC data. A sigmoid shape is observed in the QC model variation of site $\mathrm{I}^{\prime}$ population in the range of $\mathrm{CN} \sim 45-55$. It has no clear physical meaning. It is in this range of aluminum content that a reversal of site preferences is found in the QC model (see Figure 6). It is thus possible that this behavior is due to a numerical instability of the QC model equations.

The site energy differences $\left(e_{\mathrm{I}^{\prime}}-e_{\mathrm{I}}\right)$ and $\left(e_{\mathrm{II}}-e_{\mathrm{I}}\right)$ as a function of $\mathrm{CN}$ are shown in Figure 6 for the QC model and for the model used in the present simulations. A linear variation of the energy differences with $\mathrm{CN}$ is found in our simulations, in agreement with the assumption of the QC model. The origin of this finding is simply that the partial charges on the framework atoms vary linearly with $\mathrm{CN}$. 


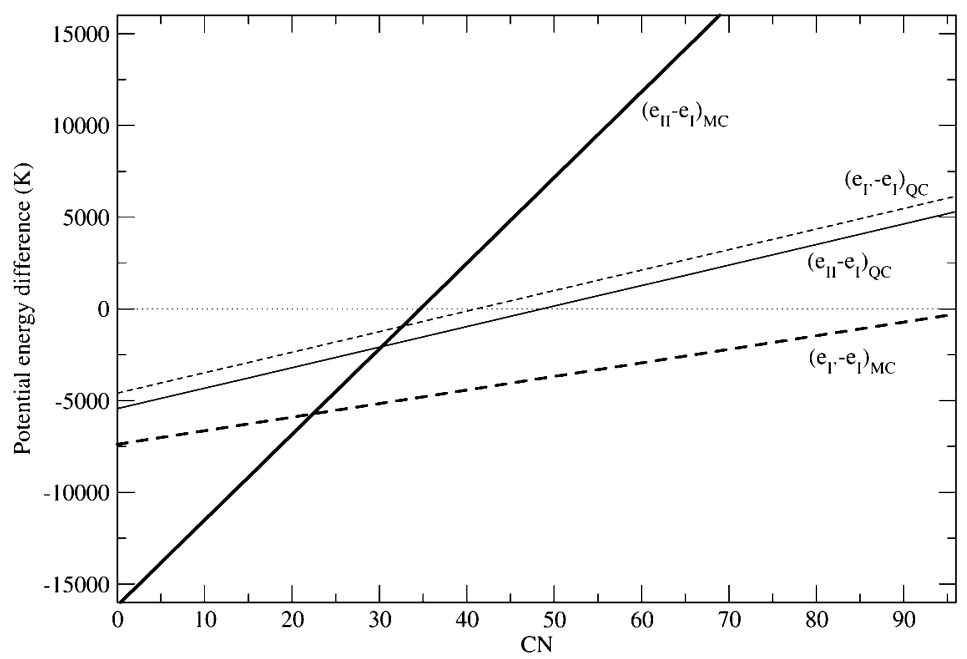

Figure 6. Sites energy differences $\left(e_{\mathrm{II}}-e_{\mathrm{I}}\right)$ and $\left(e_{\mathrm{I}^{\prime}}-e_{\mathrm{I}}\right)$ as a function of the cation number $\mathrm{CN}$ for the Monte Carlo simulations (MC) and the Quasi Chemical model (QC).

Absolute values of the energy difference in both models are not strictly comparable, because only an effective $\mathrm{I}-\mathrm{I}^{\prime}$ interaction is taken into account in the QC model, through the $w$ parameter, whereas in the simulation model, the Coulombic interaction between cations in sites I, I', II, and III is explicitly computed.

The individual site preference for low $\mathrm{CN}$ is, in both cases, II $>$ I $^{\prime}>$ I. A reversal of site preference is found as $\mathrm{CN}$ increases. At high $\mathrm{CN}$ values, the cation site preference is $\mathrm{I}^{\prime}>$ I > II for the MC model and I > II > I' for the QC model. The difference in site preference and in variation of the energy differences between the two models is quite large. However, as already pointed out by Mortier and co-workers, ${ }^{25}$ the cation occupancy scheme is dominated by the Coulombic repulsive energy between cations, at least for a high enough cation number. This explains why the differences observed in Figure 6 between the two models have no profound effect on the cation site populations. Finally, it is interesting to note that both models predict that site II will be preferentially occupied as the cation number tends to zero. This means that residual cations in dealuminated faujasite would be located in sites II rather than in sites I', which is in contradiction with conventional wisdom, as pointed out earlier by Lievens et al. ${ }^{5}$

\section{Conclusion}

We have used the Monte Carlo replica exchange (MCRE) algorithm to compute the sodium cation distribution in faujasite. The resulting distributions were found to be in very good agreement with both available experiments and the QC model of Mortier and co-workers.

The QC model has the advantage, over MC simulations, that it is simple and analytical. The assumptions of the model, however, are based on a preliminary knowledge of the cation site topology (the existence of $\mathrm{I}-\mathrm{I}^{\prime}$ nearest neighbor sites for instance). This is not the case for MC simulations, which could in principle be extended to any type of nanoporous materials, provided that the framework structure is known and that a decent cation force field is available.

The MCRE method is rather time-consuming. A drastic simplification of the method consists of using site-to-site hopping in a single $(\mathrm{N}, \mathrm{V}, \mathrm{T})$ Monte Carlo simulation at room temperature. This enables one to jump over the potential energy barriers in the configuration space and leads to a rapid convergence of the MC simulations. We have checked here that site-to-site hopping simulations yield essentially identical results as the more general MCRE method.

The main advantage of the MCRE method is that no assumption is needed on the actual cation adsorption sites. One could thus, in principle, predict cation locations and distributions in a zeolite for which the precise location of extraframework cations is not known.

We intend to implement this method into multicomponent adsorption calculations, in which at least one of the adsorbates is a polar molecule. Recently, Mellot and Cheetham ${ }^{47}$ have carried out a neutron scattering study of $\mathrm{CFCl}_{3}$ adsorption in $\mathrm{NaY}$. Cation redistribution was observed together with a new and previously unknown cation location. This is a typical situation in which the MCRE method could be useful, either for predicting cation distributions or for interpreting the experimental results.

Acknowledgment. We acknowledge Florent Calvo, Wilfried Mortier, Juan de Pablo, and Pascal Pernot for fruitful discussions.

\section{References and Notes}

(1) Mortier, W. J. Compilation of Extraframework Sites in Zeolites; Butterworth Scientific Ltd.: Guildford, U.K., 1982.

(2) Eulenberger, G. R.; Shoemaker, D. P.; Keil, J. G. J. Phys. Chem. 1967, 71, 1812.

(3) Jiràk, Z.; Vratislav, S.; Bosàcek, V. J. Phys. Chem. Solids 1980, $41,1089$. 1311.

(4) Fitch, A. N.; Jobic, H.; Renouprez, A. J. Phys. Chem. 1986, 90,

(5) Lievens, J. L.; Mortier, W. J.; Chao, K.-J. J. Phys. Chem. Solids 1992, 53, 1163.

(6) Marra, G. L.; Fitch, A. N.; Zecchina, A.; Ricchiardi, G.; Salalaggio, M.; Bordera, S.; Lamberti, C. J. Phys. Chem. B 1997, 101, 10653.

(7) Hseu, T. D. Ph.D. Thesis, University of Washington, 1972. University Microfilms, No 73-13835, Ann Arbor, Michigan.

(8) Gallezot, P.; Imelik, B. J. Chim. Phys., Physico-Chim. Biol. 1975, 68,816 .

(9) Smolin, Y. I.; Shepelev, Y. F.; Butikova, I. K.; Petranovskii, V. P. Soviet Phys. Crystallogr. 1983, 28, 36.

(10) Van Dun, J. J.; Dhaeze, K.; Mortier, W. J.; Vaughan, D. E. W. J. Phys. Chem. Solids 1989, 50, 469.

(11) Mellot, C. F. Ph.D. Thesis, Université Pierre et Marie Curie, Paris, 1993.

(12) Olson, D. H. Zeolites 1995, 15, 439

(13) Vitale, G.; Mellot, C. F.; Bull, L. M.; Cheetham, A. K. J. Phys. Chem. B 1997, 101, 4559.

(14) Plevert, J.; DiRenzo, F.; Fajula, F.; Chiara, G. J. Phys. Chem. 1997, 101,10340 .

(15) Feuerstein, M.; Lobo, R. F. Chem. Mater. 1998, 10, 2197. 
(16) Zhu, L.; Seff, K. J. J. Phys. Chem. B 1999, 103, 9512.

(17) Porcher, F.; Souhassou, M.; Dusausoy, Y.; Lecomte, C. Eur. J. Mineral. 1999, 11, 333

(18) Feuerstein, M.; Hunger, M.; Engelhardt, G.; Amoureux, J. P. Solid State Nucl. Magn. Reson. 1996, 7, 95.

(19) Plevert, J.; DeMenorval, L. C.; DiRenzo, F.; Fajula, F. J. Phys. Chem. B 1998, 102, 3412.

(20) Feuerstein, M.; Lobo, R. F. Chem. Commun. 1998, 1647.

(21) Lim, K. H.; Grey, C. P. J. Am. Chem. Soc. 2000, 122, 9768.

(22) Dempsey, E. J. Phys. Chem. 1969, 73, 3660.

(23) Sanders, M. J.; Catlow, C. R. A.; Smith, J. V. J. Phys. Chem. 1984 $88,2796$.

(24) Barrer, R. M. Zeolites 1984, 4, 361.

(25) Van Dun, J. J.; Mortier, W. J. J. Phys. Chem. 1988, 92, 6740.

(26) Van Dun, J. J.; Dhaeze, K.; Mortier, W. J. J. Phys. Chem. 1988 $92,6747$.

(27) Smolders, E.; Van Dun, J. J.; Mortier, W. J. J. Phys. Chem. 1991, 95, 9908 .

(28) Mellot, C. F.; Cheetham, A. K. C. R. Acad. Sci. Ser. IIc: Chim. 1998, 737 .

(29) Jaramillo, E.; Auerbach, S. M. J. Phys. Chem. B 1999, 103, 9589.

(30) Buttefey, S.; Boutin, A.; Mellot-Drazniecks, C. F.; Fuchs, A. H. J. Phys. Chem. B 2001, 105, 9569.

(31) Mellot-Drazniecks, C. F.; Buttefey, S.; Boutin, A.; Fuchs, A. H. Chem. Commun. 2001, 2200.

(32) Fuchs, A. H.; Cheetham, A. K. J. Phys. Chem. B 2001, 105, 7375.

(33) Talu, O. Adv. Colloid Interface Sci. 1998, 76, 227.

(34) Beerdsen, E.; Smit, B.; Calero, S. J. Phys. Chem. B 2002, 106, 10659 .
(35) Grey, C. P.; Poshni, F. I.; Gualtieri, A. F.; Norby, P.; Hanson, J. C.; Corbin, D. R. J. Am. Chem. Soc. 1997, 119, 1981.

(36) Pichon, C.; Méthivier, A.; Simonot-Grange, M.-H.; Baerlocher, C. J. Phys. Chem. B 1999, 103, 10197.

(37) Jaramillo, E.; Grey, C. P.; Auerbach, S. M. J. Phys. Chem. B 2001, $105,12319$.

(38) Marinari, E.; Parisi, G. Europhys. Lett. 1992, 19, 451.

(39) Tesi, M. C.; van Rensburg, E. J. J.; Orlandini, E.; Whittington, S. G. J. Stat. Phys. 1996, 82, 155.

(40) Hansmann, U. H. E. Chem. Phys. Lett. 1997, 281, 140.

(41) Falcioni, M.; Deem, M. W. J. Chem. Phys. 1999, 110, 1754.

(42) Yan, Q.; de Pablo, J. J. J. Chem. Phys. 1999, 116, 5419.

(43) Uytterhoeven, L.; Dompas, D.; Mortier, W. J. J. Chem. Soc., Faraday Trans. 1992, 88, 2753.

(44) Buttefey, S.; Boutin A.; Fuchs, A. H. Mol. Simul. 2002, 28, 1049.

(45) Kofke, D. J. Chem. Phys. 2002, 117, 6911.

(46) Mortier, W. J.; Van den Bossche, E.; Uytterhoeven, J. B. Zeolites 1984, 4, 41 .

(47) Mellot-Draznieks, C.; Rodriguez-carjaval, J.; Cox, D. E.; Cheetham, A. K. Phys. Chem. Chem. Phys. 2003, 5, 1882.

(48) Lignieres, J.; Newsam, J. M. Microporous Mesoporous Mater. 1999, 28, 305 .

(49) Guliants, V. V.; Mullhaupt, J. T.; Newsam, J. M.; Gorman, A. M.; Freeman, C. M. Catal. Today 1999, 50, 661.

(50) Newsam, J. M.; Freeman, C. M.; Gorman, A. M.; Vessal, B. Chem. Commun. 1996, 16, 1945. 